\title{
Effect of canagliflozin on circulating active GLP-1 levels in patients with type 2 diabetes: a randomized trial
}

\author{
Kohzo Takebayashi, Kenji Hara, Tomoko Terasawa, Rika Naruse, Mariko Suetsugu, Takafumi Tsuchiya \\ and Toshihiko Inukai
}

Department of Internal Medicine, Dokkyo Medical University Koshigaya Hospital, Koshigaya, Japan

\begin{abstract}
Canagliflozin has a robust inhibitory effect on sodium glucose transporter (SGLT)-2 and a mild inhibitory effect on SGLT1. The main purpose of this study was to investigate the effect of canagliflozin on circulating active glucagon-like peptide 1 (GLP-1) levels in patients with type 2 diabetes. Patients were randomly divided into a control group $(\mathrm{n}=15)$ and a canagliflozin-treated group $(\mathrm{n}=15)$. After hospitalization, the canagliflozin-treated group took $100 \mathrm{mg} /$ day canagliflozin for the entire study, and after 3 days both groups took $20 \mathrm{mg}$ /day teneligliptin for an additional 3 days. In a meal test, canagliflozin significantly decreased the area under curve (AUC) (0-120 min) for plasma glucose (PG) after 3 days when compared with that at baseline, and addition of teneligliptin to the canagliflozin-treated group further decreased it. A significant decrease in the AUC (0-120 min) for serum insulin by canagliflozin was obtained, but the addition of teneligliptin elevated the AUC, and thus abolished the significant difference from baseline. A significant increase in the AUC (0-120 min) of plasma active GLP-1 by canagliflozin-treatment compared with that at baseline was observed, and the addition of teneligliptin resulted in a further increase. However, canagliflozin-treatment did not change the AUC (0-120 $\mathrm{min}$ ) of plasma active glucose-dependent insulinotropic peptide (GIP). In conclusions, canagliflozin-administration before meals decreased PG and serum insulin, and increased plasma active GLP-1 levels in patients with type 2 diabetes. Canagliflozin did not greatly influence plasma active GIP levels.
\end{abstract}

Key words: Canagliflozin, GLP-1, GIP, SGLT1

SODIUM GLUCOSE COTRANSPORTER 2 (SGLT2) inhibitors are a new class of anti-diabetic drugs that improve glycemic control by inhibiting reabsorption of glucose filtered through the renal glomerulus. SGLT2 is expressed in the S1 segment of the proximal renal tubules, and inhibition of this molecule results in a remarkable increase in urinary glucose excretion [1-4]. SGLT2 is generally responsible for approximately $90 \%$ of the glucose absorption in the kidney. On the other hand, SGLT1 is expressed in the S3 segment of the proximal renal tubes, which

Submitted Feb. 14, 2017; Accepted Jun. 12, 2017 as EJ17-0065 Released online in J-STAGE as advance publication Aug. 18, 2017 Correspondence to: Kohzo Takebayashi, M.D., Department of Internal Medicine, Dokkyo Medical University Koshigaya Hospital, 2-1-50, Minami-Koshigaya, Koshigaya, Saitama 3438555, Japan. E-mail: takeb@gmail.plala.or.jp

Clinical Trial Registration: UMIN000016539 (https://upload.umin. ac.jp/cgi-openbin/ctr/ctr.cgi?function=brows\&action=brows\&type $=$ summary\&recptno $=\mathrm{R} 000019197 \&$ language $=\mathrm{E}$ ). Trial registration name: The effect of canagliflozin on active GLP-1 levels and betatrophin in patients with type 2 diabetes (CANARIA-STUDY). is located in a more distal region from the glomerulus compared with the location of S1. SGLT1 is responsible for approximately $10 \%$ of the glucose absorption in the kidney [2]. Among the SGLT2 inhibitors, canagliflozin also possesses a mild SGLT1 inhibitory effect $[5,6]$. Because SGLT1 is also expressed in the small intestine [7,8], inhibition of SGLT1 appears to cause the inhibition of glucose absorption in this location as well. In fact, it is reported that administration of canagliflozin can transiently repress the increase of postprandial plasma glucose (PG) levels probably via SGLT1 inhibition in the small intestine by a maximum of $30 \%$ in healthy subjects [9].

Interestingly, recent animal studies showed that administration of canagliflozin not only caused an increase in the glucose concentration in the lower small intestinal lumen probably by SGLT1 inhibition, but also resulted in a tendency toward an increase in the levels of plasma glucagon-like peptide (GLP)-1 (a hormone that increases insulin secretion in pancreatic $\beta$ cells) which is secreted by glucose-stimulation in $\mathrm{L}$ 
cells located mainly in the mucosal membrane of the lower small intestine [10]. The remarkable elevation of plasma active GLP-1 levels was noted especially with co-administration of dipeptidyl peptidase 4 (DPP4) inhibitors, which block GLP-1 degradation [11]. Although a tendency toward an increase in circulating active GLP-1 by canagliflozin is reported also in healthy subjects [9], there is as yet no evidence demonstrating the effect of canagliflozin on circulating active GLP-1 in patients with type 2 diabetes. Since it is known that glucose-absorption in the small intestine via SGLT1 in patients with type 2 diabetes generally increases relative to that in non-diabetic subjects [12], the possible effect of canagliflozin via SGLT1 inhibition on the circulating active GLP-1 levels may be greatly enhanced in patients with type 2 diabetes. Thus, canagliflozin may potentially have an additional beneficial effect in elevating circulating active GLP-1 levels for glycemic control in patients with type 2 diabetes.

In the current randomized open label study, we investigated the effect of canagliflozin on circulating active GLP-1 levels in patients with poorly controlled type 2 diabetes to better understand the mechanisms involved in its glycemic regulation. We hypothesized that canagliflozin increases circulating active GLP-1 levels in these patients.

\section{Patients and Methods}

\section{Patients}

The sample size was determined based on the effect of canagliflozin on circulating active GLP-1 levels in a previous report involving healthy subjects. We predicted that canagliflozin could increase active GLP-1 levels at least to the same degree in patients with type 2 diabetes, compared with that observed in healthy subjects [9]. We hypothesized that canagliflozin can achieve a maximal $1.6 \mathrm{pmol} / \mathrm{L}$ increase (standard deviation $1.1 \mathrm{pmol} / \mathrm{L}$ ) in active GLP-1 levels. A sample size of 12 per group would be the minimum required under the conditions of a power of $90 \%$, an $\alpha$ of 0.05 two-tailed, and a dropout rate of $20 \%$.

The study, named CANARIA (the effect of CANAgliflozin on active GLP-1 levels and betatRophin in patients with type 2 dIAbetes), was performed from February 2015 to December 2015 at Dokkyo Medical University Koshigaya Hospital (Koshigaya, Japan). Although the original protocol also included the measurement of betatrophin, which is a hormone associated with lipid metabolism, we have focused on the effect of canagliflozin on GLP-1 levels in this paper. The initial target number of recruited patients was 40 , and during the study period, 39 patients were enrolled. We decided to conduct the study with 39 enrolled patients. This study was registered at UMIN 000016539. The patients were randomly assigned to two groups [canagliflozin-treated group $(n=19)$ and control group $(\mathrm{n}=20)]$, by a central computer system based on hemoglobin A1c (HbAlc) levels, body mass index (BMI), gender, and age.

Key inclusion criteria were 1) patients (male or female) aged 20 or over, 2) patients diagnosed as having type 2 diabetes (based on the criteria of the Japan Diabetes Society 2010) [13], 3) patients with diet and exercise therapy for diabetes for 12 weeks or more prior to starting the study (if patients have severe diabetic complications, diet therapy alone was acceptable), 4) patients who had not changed the type and dose of all anti-diabetic drugs for the 4 weeks prior to starting the study, 5) patients who had not taken DPP4 inhibitors, GLP-1 receptor agonists, and SGLT2 inhibitors for 1 month prior to starting the study, 6) patients with $\mathrm{HbA1c}$ levels more than or equal to $6.5 \%$ at enrollment , 7) patients who were hospitalized for glycemic control for type 2 diabetes, and 8) patients who provided consent in writing for participation in this study. Key exclusion criteria were 1) patients with either type 1 diabetes, pancreatic diabetes, secondary diabetes (Cushing syndrome, acromegaly etc.), diabetic coma, or diabetic pre-coma, 2) patients who were not candidates for either canagliflozin or teneligliptin treatment, 3) patients taking insulin, 4) heavy alcohol consumption (more than or equal to $60 \mathrm{~g}$ /day of pure alcohol), 5) patients who were either pregnant, lactating, or had the chance of becoming pregnant, 6) patients with severe renal dysfunction (serum creatinine $>2.4 \mathrm{mg} / \mathrm{dL}$ or creatinine clearance $<30 \mathrm{~mL} / \mathrm{min}$ ), and 7) patients judged as inadequate for participation in this study by a medical doctor.

Clinical and laboratory tests showed no evidence of either moderate or severe liver dysfunction or autoimmune disease in any patient. No patients had serum creatinine levels exceeding $1.1 \mathrm{mg} / \mathrm{dL}$ except for 2 patients in the canagliflozin-treated group (1.28 and $1.51 \mathrm{mg} /$ dL). After enrollment, all patients were hospitalized during the study-period. Four patients in the control group and 2 in the canagliflozin-treated group retracted their written agreement on participation at least before initiation of the study (i.e., the Meal test). One patient 
in the canagliflozin-treated group was released from the study because the patient had type 1 diabetes on admission. One patient in the control group and 1 in canagliflozin-treated group were excluded because they had received co-treatment with a DPP4 inhibitor just prior to hospitalization. As a result, 15 patients in the control group and 15 in the canagliflozin-treated group completed the study.

The characteristics of the patients in the canagliflozin-treated group and in the control group on enrollment are shown in Table 1.

\section{Methods}

All data in this study was collected in our hospital. This study was a randomized, parallel-group comparison, open label study. This was not a double-blind study, because the use of a placebo and a double-blind design are not usually permitted as a post-marketing clinical study in Japan. In addition, SGLT2 inhibitors appears not to be suitable for a double-blind design because of clinical symptoms, such as polyuria and an increase in glucose in the urine. The ratio of assignment between the groups with and without study drug was $1: 1$. The randomization was performed using the minimization method by a company, Medical Edge Co., Ltd, Tokyo, Japan. The concrete computer-system for the assignment was designed by the company. The enrollment of the patients was performed by each medical doctor in charge who participated in this study.

On day 2 (the next day after hospitalization), patients in the canagliflozin-treated group took canagliflozin
(100 mg, $1 \mathrm{Tab}$ ) after the first meal test (before lunch). Thereafter, patients in this group took canagliflozin 30 min before eating breakfast (or test meal) in all days since next day (day 3). On day 5, patients in both groups took teneligliptin (20 mg, $1 \mathrm{Tab}$ ) (a DPP4 inhibitor) after finishing the second meal test (before lunch). Since day 6, patients in the control group took teneligliptin only, and those in the canagliflozin group took both canagliflozin and teneligliptin simultaneously 30 min before breakfast (or meal test).

\section{Meal Test}

The commercially available test meal, Meal test C (cookie type) (Saraya Co, Ltd, Osaka, Japan) was used. After overnight fast of at least $10 \mathrm{hr}$ (from $2000 \mathrm{~h}$ the previous day), each patient took one container of Meal test $\mathrm{C}$ basically at $0900 \mathrm{~h}$ on days 2,5 , and 8 after hospitalization. The cookie (1 container) consisted of 75 $\mathrm{g}$ of carbohydrate ( $0 \mathrm{~g}$ of sucrose), $28.5 \mathrm{~g}$ of fat, 8.0 $\mathrm{g}$ of protein, $0.5-4.0 \mathrm{~g}$ of dietary fiber, and $125 \mathrm{mg}$ of sodium yielding $592 \mathrm{kcal}$ as total calories. Patients were required to take all test meals with some water at least within $15 \mathrm{~min}$ of the test. Blood was collected at 0 (just before eating), 30, 60, and $120 \mathrm{~min}$ on the days of the three meal tests (days 2, 5, and 8).

\section{Measurement of active GLP-1 and GIP levels}

For measuring the plasma active GLP-1 and GIP levels, blood was collected into commercial test tubes specific for these measurements containing EDTA2K and DPP4 inhibitor etc., (BDTM P800, Becton, Dickinson and Company, NJ, USA). Active GLP-1 and active GIP levels were measured

Table 1 Clinical characteristics of the patients treated without and with canagliflozin

\begin{tabular}{|c|c|c|c|}
\hline & Control & Canagliflozin 95\%CI & $p$ \\
\hline No. (male/female) & $15(9 / 6)$ & $15(11 / 4)$ & - \\
\hline Age (year) & $59.4 \pm 12.5$ & $57.9 \pm 10.6(-7.2,10.1)$ & 0.7316 \\
\hline BMI $\left(\mathrm{kg} / \mathrm{m}^{2}\right)$ & $24.6 \pm 4.9$ & $26.5 \pm 5.1(-5.5,1.9)$ & 0.3305 \\
\hline FPG (mg/dL) & $176.5 \pm 54.4$ & $174.7 \pm 71.5(-45.8,49.3)$ & 0.9410 \\
\hline $\mathrm{HbAlc}(\%)$ & $10.5 \pm 2.8$ & $10.4 \pm 1.7(-1.7,1.9)$ & 0.8964 \\
\hline \multicolumn{4}{|l|}{ Diabetic therapy } \\
\hline $\mathrm{S} / \mathrm{M} / \mathrm{G} / \mathrm{P} / \mathrm{S}+\mathrm{M} / \mathrm{A} / \mathrm{S}+\mathrm{M}+\mathrm{A}$ & $2 / 4 / 0 / 0 / 2 / 2 / 1$ & $1 / 5 / 1 / 1 / 1 / 0 / 2$ & - \\
\hline $\mathrm{S}+\mathrm{A}+\mathrm{P} / \mathrm{S}+\mathrm{M}+\mathrm{P} / \mathrm{S}+\mathrm{P}$ & $1 / 0 / 0$ & $1 / 2 / 1$ & - \\
\hline $\mathrm{G}+\mathrm{P} / \mathrm{N}$ & $1 / 2$ & $0 / 0$ & - \\
\hline \multicolumn{4}{|l|}{ Antihypertensive drugs } \\
\hline $\mathrm{Ar} / \mathrm{C} / \mathrm{Ar}+\mathrm{C} / \mathrm{Ar}+\mathrm{T} / \mathrm{Ar}+\mathrm{C}+\mathrm{T} / \mathrm{Ai}$ & $1 / 2 / 4 / 1 / 0 / 1$ & $3 / 2 / 0 / 0 / 2 / 0$ & - \\
\hline Statins & 9 & 7 & \\
\hline
\end{tabular}

Data are expressed as mean \pm standard deviation (SD). $p: p$ value by unpaired $t$ test. $95 \% \mathrm{CI}, 95 \%$ confidence interval for the difference; BMI, body mass index; FPG, fasting plasma glucose; HbAlc, hemoglobin Alc. Diabetic therapy: the number of the patients with respective diabetic therapies. S, sulfonylureas; M, metformin; G, glinides; P, pioglitazone; A, $\alpha$ glucosidase inhibitors; N, no antidiabetic drugs. Antihypertensive drugs: the number of the patients with respective antihypertensive drugs. Ar, angiotensin-II receptor blockers (ARB); $\mathrm{C}$, calcium channel blockers; T, thiazides; Ai, angiotensin converting enzyme inhibitors (ACE-I). 
after a solid-phase extraction using a Glucagon-Like Peptide-1 (active) Enzyme-Linked ImmunoSorbent Assay (ELISA) kit (EGLP-35K) (Merck KGaA, Ltd., Darmstadt, Germany) and using a Human GIP, Active form Assay Kit-IBL (sandwich ELISA kit) (Immuno-Biological Laboratories Co., Ltd, Gunma, Japan). The lower limit of detection of active GLP-1 with this kit was $2 \mathrm{pmol} / \mathrm{L}$, and if the value was less than $2 \mathrm{pmol} / \mathrm{L}$, the data were defined as $2 \mathrm{pmol} / \mathrm{L}$. The intraassay and interassay CV of active GLP-1 was 6.44$8.51 \%$ and $5.87-9.85 \%$, respectively, and that of active GIP was 3.26-6.18\% and 1.83-8.91\%, respectively.

\section{Measurement of Urinary $C$ peptides}

Urinary C peptides were measured using a 24-h urine collection starting on the day of admission as a rule. The $\mathrm{C}$ peptides were measured by a chemiluminescent Enzyme Immunoassay (CLEIA) using the agent LUMIPULSE ${ }^{\circledR}$ C-peptide (FUJIREBIO Inc., Tokyo, Japan).

\section{Diet and Exercise}

While hospitalized, the patients received diet therapy determined approximately by the following formula: total food requirement $=$ height $(\mathrm{m})^{2} \times 22 \times 25$ (kcal/day). Patients were asked to walk an additional $20 \mathrm{~min}$ at normal speed in addition to the usual walking in the hospital. This additional walking was considered comparable to the amount of physical exertion of 1 metabolic equivalent $(\mathrm{MET}) \cdot \mathrm{h}$. The exact total amount of physical exertion per day by each patient was not estimated in this study.

\section{Ethical Considerations}

All subjects provided written informed consent for inclusion in the study. The study (study no. 1433) was approved by the Local Ethics Committee at our hospital and was performed according to the guidelines of the Declaration of Helsinki.

\section{Statistical Methods}

The two time points for each parameter, excluding aspartate transaminase (AST), alanine transaminase (ALT), gamma-glutamyl transpeptidase (GGT), and high sensitivity $\mathrm{C}$ reactive protein (hsCRP), for an individual were compared using a paired $t$-test because all data, excluding the above variables, followed a normal distribution as confirmed by a $\chi^{2}$ goodness of fit test. For these variables, the Wilcoxon signed rank test was used because of the skewed distribution. A comparison of various markers, excluding AST, ALT, GGT, and hsCRP, for changes from baseline between control and canagliflozin-treated patients was assessed using an analysis of covariance (ANCOVA) model with treatment as the fixed effect and the corresponding baseline value as a covariate. For these comparisons involving AST, ALT, GGT, and hsCRP, the Mann-Whitney U test was used. For the comparison among 3 time points, a parametric comparison was performed using a one-way repeated measures analysis of variance (ANOVA). If the ANOVA was significant, a Holm test was used as a post hoc test. However, because of the potentially skewed distributions of the AUC (0-120 min) of active GLP-1 at baseline in the canagliflozin-treated group, the comparison for the AUC (0-120 min) of active GLP-1 among 3 time points in this group was also confirmed by the Friedman test accompanied with a post hoc test by the Scheffe test as a non-parametric analysis. For the comparison between control and canagliflozin-treated groups for the difference between the AUC (0-120 min) at 3 days after baseline (day 5) and that at baseline (day 2) on either active GLP-1 or GIP, and for the similar comparison at 6 days after baseline (day 8) and at baseline on active GIP, an unpaired $t$ test was used because the data followed a normal distribution as confirmed by a $\chi^{2}$ goodness of fit test. Based on the evaluation of equality of variances confirmed by the F test, either the Student's $t$ test or the Welch's $t$ test was chosen. For the comparison between 2 groups for the difference between the AUC $(0-120 \mathrm{~min})$ at 6 days after baseline and that at baseline on active GLP-1, the Mann-Whitney U test was chosen because of the skewed distribution for the difference between the AUC (0-120 min) at 6 days after baseline and that at baseline on active GLP-1 in the control group. All statistical analyses were performed using Ekuseru-Toukei 2012 software (Social Survey Research Information Co., Ltd, Tokyo, Japan). A $p$ value of less than 0.05 was accepted as indicating statistical significance (two-sided).

\section{Results}

No adverse events were observed during the study. On admission, urinary ketone body levels were negative in all patients.

Changes in several variables at baseline (day 2) and after 3 days (day 5) in both control and canagliflozin-treated groups are presented in Table 2. The effect of both canagliflozin and teneligliptin on PG, serum insulin (immunoreactive insulin), 
Table 2 Changes in various variables at baseline and 3 days after therapy

\begin{tabular}{|c|c|c|c|c|c|c|c|}
\hline & \multicolumn{3}{|c|}{ Control } & \multicolumn{3}{|c|}{ Canagliflozin } & \multirow{2}{*}{$p^{*}$} \\
\hline & Baseline & 3 days & $p$ (Difference: $95 \% \mathrm{CI})$ & Baseline & 3 days & $p$ (Difference: $95 \% \mathrm{CI})$ & \\
\hline FPG $(\mathrm{mg} / \mathrm{dL})$ & $176.5 \pm 54.4$ & $169.1 \pm 61.0$ & $0.3646(-9.4,24.1)$ & $174.7 \pm 71.5$ & $137.7 \pm 50.1$ & $0.0006(18.9,55.0)^{*}$ & $0.0083^{*}$ \\
\hline $\mathrm{TC}(\mathrm{mg} / \mathrm{dL})$ & $185.6 \pm 34.9$ & $180.0 \pm 30.3$ & $0.3623(-6.6,17.0)$ & $183.7 \pm 30.0$ & $192.0 \pm 35.5$ & $0.1218(-19.0,2.5)$ & 0.7862 \\
\hline $\mathrm{TG}(\mathrm{mg} / \mathrm{dL})$ & $141.7 \pm 57.7$ & $147.7 \pm 51.7$ & $0.6146(-30.6,18.8)$ & $133.9 \pm 58.5$ & $120.7 \pm 45.7$ & $0.2995(-13.1,39.5)$ & $0.0009 *$ \\
\hline HDL-C (mg/dL) & $54.1 \pm 13.6$ & $52.3 \pm 14.1$ & $0.1174(-0.5,4.1)$ & $45.5 \pm 12.1$ & $44.8 \pm 12.7$ & $0.5132(-1.5,2.8)$ & 0.9101 \\
\hline LDL-C (mg/dL) & $107.6 \pm 36.4$ & $103.9 \pm 32.9$ & $0.5352(-8.7,16.3)$ & $116.5 \pm 25.3$ & $125.7 \pm 33.0$ & $0.0721(-19.5,1.0)$ & 0.1882 \\
\hline $\mathrm{UA}(\mathrm{mg} / \mathrm{dL})$ & $5.3 \pm 1.3$ & $5.4 \pm 1.8$ & $0.5586(-0.6,0.3)$ & $5.8 \pm 1.5$ & $6.0 \pm 1.9$ & $0.6609(-0.9,0.5)$ & 0.7599 \\
\hline $\mathrm{Cr}(\mathrm{mg} / \mathrm{dL})$ & $0.75 \pm 0.12$ & $0.78 \pm 0.14$ & $0.0826(-0.07,0.01)$ & $0.79 \pm 0.30$ & $0.87 \pm 0.33$ & $0.0030(-0.13,-0.03)^{*}$ & 0.3980 \\
\hline AST (U/L) & $19(17.5,2.2)$ & $20(16.5,22.5)$ & 0.3882 & $18(15.5,43)$ & $24(16,41)$ & 0.9750 & 0.9502 \\
\hline $\operatorname{ALT}(\mathrm{U} / \mathrm{L})$ & $19(18,35)$ & $20(17.5,33)$ & 0.4777 & $28(16,43.5)$ & $31(15,45.5)$ & 0.9547 & 0.5729 \\
\hline GGT (U/L) & $28(19.5,49)$ & $27(18.5,48.5)$ & 0.5525 & $40(18.5,58.5)$ & $38(18,56.5)$ & 0.5294 & 0.8669 \\
\hline Ht $(\%)$ & $40.9 \pm 12.1$ & $40.1 \pm 11.8$ & $0.6904(-1.3,18)$ & $42.3 \pm 4.2$ & $43.0 \pm 4.3$ & $0.1230(-1.5,0.2)$ & 0.2733 \\
\hline hsCRP (mg/L) & $\begin{array}{c}0.116 \\
(0.059,0.235)\end{array}$ & $\begin{array}{c}0.102 \\
(0.047,0.1895)\end{array}$ & 0.0901 & $\begin{array}{c}0.104 \\
(0.060,0.253)\end{array}$ & $\begin{array}{c}0.122 \\
(0.041,0.173)\end{array}$ & 0.6293 & 0.1111 \\
\hline $\mathrm{SBP}(\mathrm{mm} \mathrm{Hg})$ & $121.1 \pm 21.3$ & $115.5 \pm 21.7$ & $0.2813(-5.1,16.3)$ & $124.5 \pm 16.0$ & $121.4 \pm 12.6$ & $0.5675(-8.3,14.6)$ & $0.0008^{*}$ \\
\hline DBP (mm Hg) & $70.7 \pm 15.0$ & $67.4 \pm 12.4$ & $0.3458(-3.9,10.7)$ & $69.9 \pm 12.3$ & $73.7 \pm 6.3$ & $0.2857(-11.3,3.6)$ & $<0.0001^{*}$ \\
\hline BW (kg) & $62.6 \pm 14.0$ & $61.9 \pm 13.7$ & $0.0030(0.3,1.2)^{*}$ & $70.3 \pm 16.4$ & $69.1 \pm 16.0$ & $<0.0001(0.8,1.6)^{*}$ & $0.0141^{*}$ \\
\hline
\end{tabular}

$p: p$ value for change before and after therapy by paired $t$ test excluding AST, ALT, GGT and hsCRP. For AST, ALT, GGT and hsCRP, $p$ means the $p$ value by Wilcoxon signed rank test. $p^{*}: p$ value for comparisons in control and canagliflozin-treated groups for changes from baseline assessing using analysis of variance (ANCOVA) model with treatment as fixed effects and corresponding baseline value as covariate, excluding AST, ALT, GGT and hsCRP. For AST, ALT, GGT and hsCRP, $p^{*}$ means the $p$ value by Mann-Whitney U test. $p$ and $p *<0.05$ are defined as statistically significant $(*) .95 \% \mathrm{CI}, 95 \%$ confidence interval for the difference; Data are expressed as means \pm SD excluding AST, ALT, GGT and hsCRP. Data for AST, ALT, GGT and hsCRP are expressed as median (25 percentile, 75 percentile). FPG, fasting plasma glucose; TC, total cholesterol; TG, triglyceride; HDL-C, high-density lipoprotein cholesterol; LDL, low-density lipoprotein cholesterol; UA, uric acid; Cr, creatinine; AST, aspartate transaminase; ALT, alanine transaminase; GGT, gamma-glutamyl transpeptidase; Ht, hematocrit; hsCRP, high sensitivity $\mathrm{C}$ reactive protein; SBP, systolic blood pressure; DBP, diastolic blood pressure; BW, body weight.

plasma active GLP-1, and plasma active GIP levels by the meal test is showed in Fig. 1. Canagliflozin significantly decreased the area under curve (AUC) (0-120 $\mathrm{min})$ for $\mathrm{PG}$, and addition of teneligliptin to the canagliflozin-treated group resulted in an additional decrease (Fig. 1D). Canagliflozin significantly decreased the AUC (0-120 min) for insulin, but the addition of teneligliptin elevated the AUC for insulin, and thus abolished the significant difference from baseline (Fig. 1H). Canagliflozin significantly increased the AUC (0-120 min) of plasma active GLP-1, and the addition of teneligliptin to the canagliflozin treatment resulted in an additional elevation (Fig. 1L). However, as described in Statistical Methods above, because of the potentially skewed distribution of the data for the AUC (0-120 min) of active GLP-1 at baseline in canagliflozin-treated group, these data were also evaluated by a non-parametric analysis. The statistical significance did not change by using a non-parametric analysis: the median values ( 25 percentile, 75 percentile) for the AUC (0-120 min) of active GLP-1 at baseline, after 3 days, and after 6 days in the canagliflozin-treated group were respectively $4.975(4.025,6.1875), 9.05(5.888,14.1)$ and 22
$(17.488,28.238) \mathrm{pmol} / \mathrm{L} \cdot \mathrm{h}$. The $p$ values for the comparison of active GLP-1 AUC (0-120 min) for baseline $v s .3$ days after baseline, baseline $v s .6$ days after baseline, 3 days vs. 6 days after baseline were respectively $0.0361,<0.0001$, and 0.0170 . The difference in the AUC (0-120 min) for active GLP-1 between 3 days after baseline and baseline, and between 6 days after baseline and baseline were significantly larger in the canagliflozin-treated group than that in the control group ( $p<0.0001, p=0.0030$, respectively) (Fig. 1Q). Although administration of canagliflozin did not change the AUC (0-120 min) for plasma active GIP for the first 3 days, the subsequent addition of teneligliptin significantly increased the AUC levels in both the control and canagliflozin-groups (Fig 1N, P). However, a transient small tendency toward a decline in active GIP levels by canagliflozin (in $30 \mathrm{~min}$ ) was noted (no statistical significance) (Fig. 1O). There was a tendency toward a lower value for the difference between the AUC (0-120 min) of active GIP at 6 days after baseline and that at baseline in the canagliflozin-treated group compared with that in the control group, although the difference did not achieve statistical significance (Fig. 1R). 
A

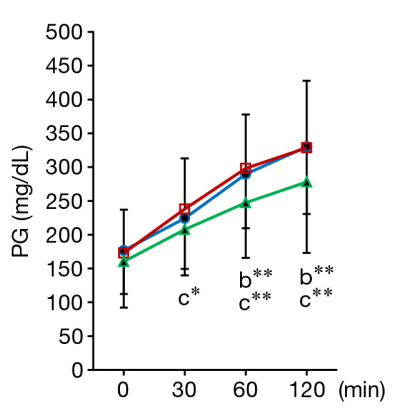

E

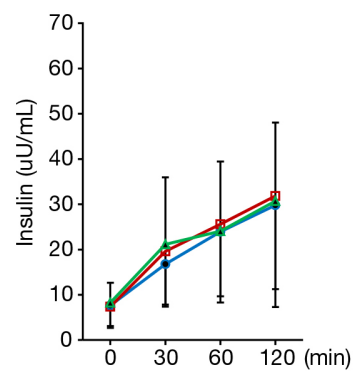

I

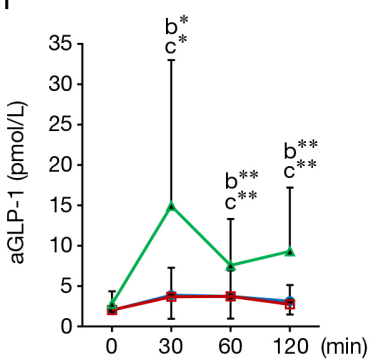

M

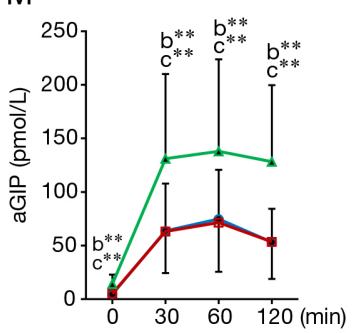

B

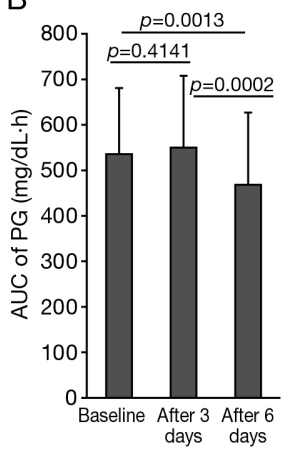

F
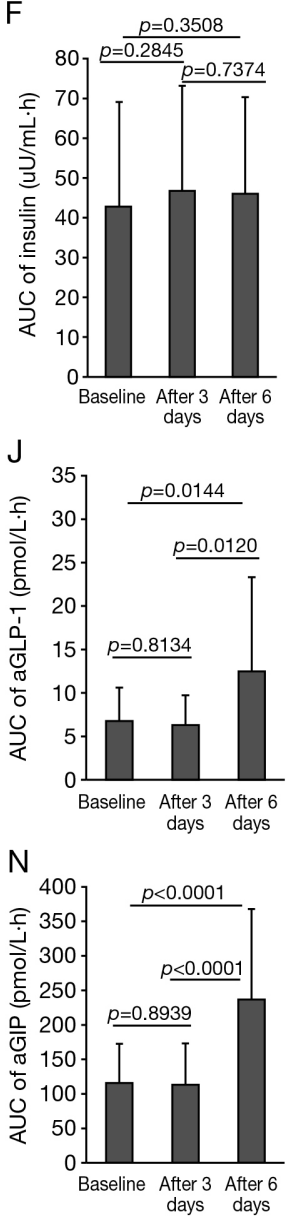

C

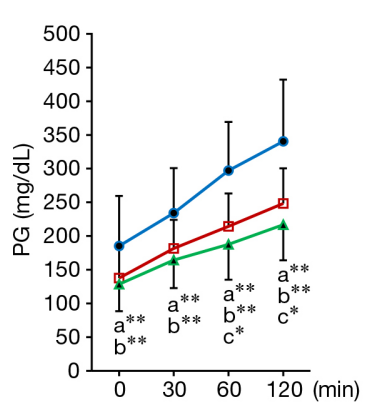

G
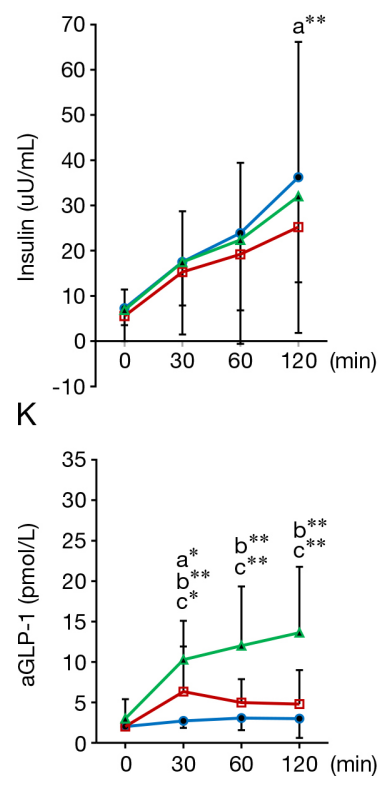

O

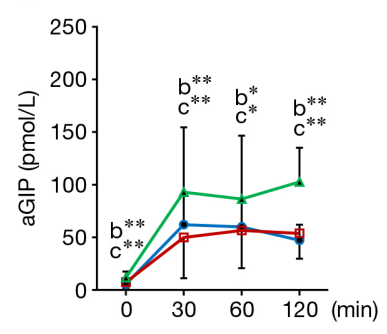

D

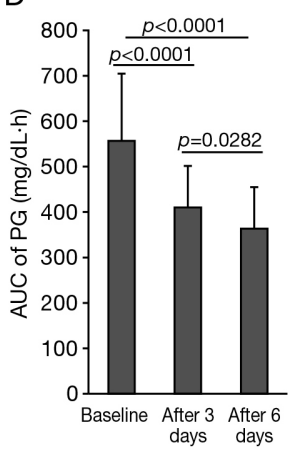

$\mathrm{H}$

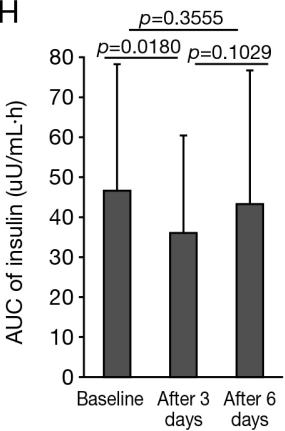

L
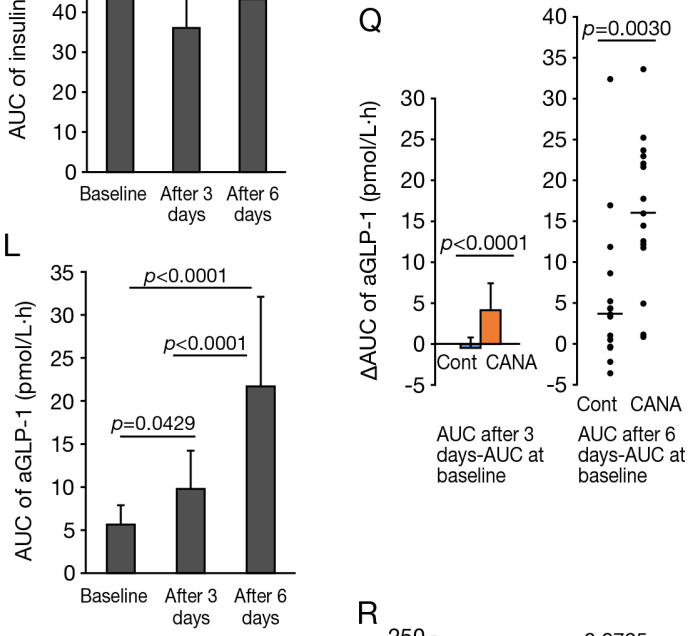

$\mathrm{P}$

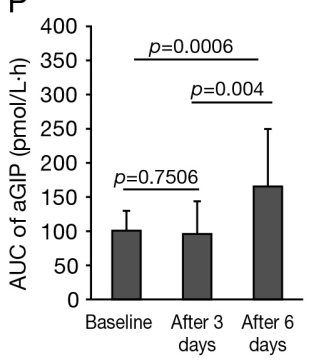

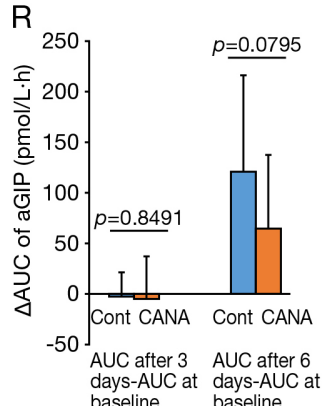

Fig. 1 Results of the meal test for PG, serum insulin, plasma active-GLP-1, and plasma active GIP levels

Bar graph and error bar respectively show mean and standard deviation. A, B, E, F, I, J, M, N: control group, C, D, G, H, K, L, $\mathbf{O}, \mathbf{P}$ : canagliflozin-treated group. A, C, E, G, I, K, M, O: Closed circle (blue line): baseline (day 2), Open square (red line): 3 days after observation without additional drugs in the control group or after addition of canagliflozin (day 5), Closed triangle (green line): 3 days after addition of either teneligliptin to the control group or addition of teneligliptin to the canagliflozin-treated group, i.e., 6 days after baseline (day 8). Q: Comparison between control and canagliflozin-treated groups for the difference in the AUC of active GLP-1 between 3 days or 6 days after baseline and baseline. For the difference between the AUC at 6 days after baseline and that at baseline on GLP-1, data are shown by scatter plots. Horizontal line shows the median value. R: Comparison between control and canagliflozin-treated groups for the difference in the AUC of active GIP between 3 days or 6 days after baseline and baseline. a: Difference between each value at baseline and after 3 days. b: Difference between each value at baseline and after 6 days. c: Difference between each value after 3 days and after 6 days. ${ }^{*} p<0.05$, and ${ }^{* *} p<0.0001$. AUC, area under the curve $(0-120$ min); aGLP-1, active GLP-1; aGIP, active GIP; Cont, control group; CANA, canagliflozin-treated group. 
The 24-h urinary $\mathrm{C}$ peptide level was measured in 29 out of the 30 patients. The $\mathrm{C}$ peptide levels in the total $(n=29)$, control $(n=14)$, and canagliflozin-treated groups $(\mathrm{n}=15)$ were respectively $74.4 \pm 44.1$ (range: 16.4-190), 90.0 \pm 50.3 (range: $22.3-190$ ), and 59.7 \pm 32.5 (range: 16.4-113) $\mu \mathrm{g} /$ day. Only 1 patient in the canagliflozin-treated group showed less than $20 \mu \mathrm{g}$ /day of urinary $\mathrm{C}$ peptide. There was no correlation between the C-peptide levels and the difference in the AUC (0-120 min) for either PG, insulin, or active GLP-1 between 3 days after baseline and baseline in the canagliflozin-treated group. On the other hand, a significant positive correlation was found between the C-peptide levels and the difference in the AUC (0-120 min) for active GIP between 3 days after baseline and baseline in this group $(\mathrm{R}=0.6472, p=0.0091)$.

\section{Discussion}

In the current study, we investigated for the first time the effect of canagliflozin on plasma active GLP-1 levels in patients with type 2 diabetes. As expected, administration of canagliflozin for 3 days significantly increased the AUC levels of active GLP-1 from baseline, and the differences in the $2 \mathrm{~h}$-AUC between 3 days after initiation of canagliflozin treatment and baseline were significantly greater than that in the control group, suggesting that canagliflozin can increase active GLP-1 plasma levels. The effect appears to be more evident in this study consisted of the patients with poorly controlled type 2 diabetes, compared with that in a previous study performed in healthy subjects [9]. The reason for this result may be based on the fact that the absorption of glucose in the small intestine is more promoted in type 2 diabetes compared with that in healthy subjects [12], and that therefore a much greater amount of glucose moves to the lower small intestine by inhibiting SGLT1 in the upper small intestine, resulting in a stronger promotion of glucose-stimulated GLP-1 secretion by L cells. Importantly, canagliflozin-treatment significantly increased active GLP-1 levels from baseline at $30 \mathrm{~min}$, suggesting that canagliflozin could elevate plasma active GLP-1 levels in the early phase. It has been reported that plasma GLP-1 levels increase from the early phase within 15-30 min with a second small peak at 90-120 min after a meal [14]. The reason for this early elevation of GLP-1 was proposed to involve indirect mechanisms, such as endocrine- or neural responses that may be needed because the meal does not arrive in such a short time in the lower small intestine, where most of the L cells are located [15]. However, recent evidence suggests that although the numbers of L cells are much higher in the lower intestine, L cells also exist in the upper intestine, which may be responsible for this rapid elevation of GLP-1 $[16,17]$. If $\mathrm{L}$ cells are present in the upper intestine, possible SGLT1 inhibition by canagliflozin could decrease (but not increase) the glucose-stimulated GLP-1 secretion via SGLT1 during the early phase. It is difficult to explain the result in this study exactly, however, there is a recent report demonstrating that empagliflozin, which is high selective for SGLT2 [5], also increased plasma GLP-1 levels although the effect appeared to be weak [18]. In that study, empagliflozin increased GLP-1 levels from the early phase just after the meal test. The effect was small but observable even before the meal test. Therefore, it is possible that the effect of canagliflozin on GLP-1 secretion might be at least partially independent of SGLT1 inhibition, especially in the early phase although the detailed mechanisms are unknown. In contrast, the possible effect of canagliflozin on inhibiting SGLT1 should be important in the late phase of GLP-1 secretion because the meal reaches the lower intestine by 2 hours, resulting in promotion of glucose-stimulated GLP-1 secretion by L cells, and because the inhibitory effect of canagliflozin on SGLT1 in L cells probably disappear in a relatively short time (within $2 \mathrm{~h}$ ) [19]. Notably, in the current study, we used $100 \mathrm{mg}$ of canagliflozin once a day unlike the previous clinical study in healthy subjects in which $300 \mathrm{mg}$ once a day was used [9] because only this dose is approved in Japan. Stein et al. reported that a dose above $150 \mathrm{mg}$ is needed to reduce plasma postprandial glucose via a non-renal mechanism (probably via inhibiting glucose absorption in the small intestine) in patients with type 2 diabetes [20]. We speculate that the reason for our positive results with this dose of canagliflozin is based, at first, on the difference in the background of patients such as race, BMI, and state of glycemic control as reflected by baseline $\mathrm{HbAlc}$ levels. In addition, an animal study showed that a relatively low dose of phlorizin (a non-selective SGLT inhibitor) did not inhibit the elevation in blood glucose levels after glucose loading, but resulted in a significant increase of GLP-1 secretion [21]. This finding might also partially explain our results. Finally, an effect independent 
of SGLT1 inhibition might have also partially influenced the results as described above. In the current study, further elevation of plasma active GLP-1 levels was observed by addition of teneligliptin, a DPP4 inhibitor, supporting the results of previous animal studies $[10,11]$. This finding appears to suggest a benefit of co-treatment of canagliflozin with DPP4 inhibitors for elevating circulating GLP-1 levels.

In the current study, we also for the first time evaluated the effect of canagliflozin on active (but not total) plasma GIP levels in patients with type 2 diabetes. Although, a transient non-significant early phase decrease was noted with canagliflozin, the total AUC of GIP by $2 \mathrm{~h}$ did not change. This finding is different from that in a previous study in healthy subjects, in which a decrease of total GIP with canagliflozin was clearly shown [9]. Although our finding might be partially due to the measurement of active (but not total) GIP, we speculate that the effect of canagliflozin on GIP may be milder when compared with that on GLP-1 in patients with type 2 diabetes but not in healthy subjects. We speculate that the possible decrease of glucose-stimulated GIP secretion due to SGLT1 inhibition of K cells (GIP secretory-cells located mainly in the mucosal membrane of the upper small intestine) [22] may not be fully realized because of the increased glucose-absorption in patients with type 2 diabetes as compared with that in healthy subjects [12]. On the other hand, the increased glucose in the intestinal lumen due to SGLT1 inhibition is probably responsible for the increase of GLP-1 secretion by L cells in the lower small intestine. However, since the tendency of a decrease in the difference between the AUC of GIP at 6 days after baseline (the time point of co-administration with teneligliptin) and that at baseline in the canagliflozin-treated group compared with that in the control group was observed, canagliflozin appears to provide a clinically apparent effect on decreasing plasma active GIP levels when it is used as a co-treatment with a DPP4 inhibitor.

In the current study, there were no correlations between the 24-h urinary C-peptide levels, which reflect endogenous insulin secretional capacity, and the change in the AUC (0-120 min) of either PG, insulin, or active GLP-1 from baseline after treatment with canagliflozin for 3 days. This may suggest that endogenous insulin secretional capacity does not influence the effect by canagliflozin on these variables. However, a positive correlation was obtained between the C-peptide levels and the change in the AUC (0-120 min) from baseline for active GIP after canagliflozin treatment for 3 days. The reason for this result is unclear. This finding will need to be confirmed in a study with a larger number of patients.

Interestingly, no insulinotropic effect was observed even after addition of teneligliptin. This may be due partially to the relative response based on the decrease in PG levels by teneligliptin. In our previous study, the administration of alogliptin (a DPP4 inhibitor) for 3 months in patients with type 2 diabetes did not change circulating insulin levels, while plasma PG were significantly decreased [23]. Notably, a homeostasis model assessment $\beta$ (HOMA- $\beta$ ), which is one of the indices for insulin secretion, was significantly improved. In contrast, HOMA-insulin resistance (IR) (an index for insulin resistance) did not change. Although the study was relatively longer (3-months) than the current study, this finding might support the lack of an effect for insulin by teneligliptin in the current study.

In the current study, treatment with canagliflozin for 3 days resulted in significant decreases in fasting PG, triglyceride, and body weight when compared with that in the control group. These findings appear to be reasonable results, given the fact that administration of SGLT2 inhibitors results in calorie loss due to the increased urinary glucose excretion. The current study period was relatively short due to ethical reasons. This is one of the limitations of this study.

\section{Conclusions}

We found that canagliflozin administration before a meal increased the plasma active GLP-1 levels, and addition of teneligliptin (a DPP4 inhibitor) further elevated these levels even at a relatively small dose (100 $\mathrm{mg}$ ) in patients with poorly controlled type 2 diabetes. Canagliflozin did not significantly change plasma active GIP levels. For understanding these findings, a longer observation period is needed.

\section{Acknowledgement}

This study was supported by funds from Mitsubishi Tanabe Pharma Co. (Osaka, Japan).

Authors' contributions: K.T. designed the study protocol. K.T., K.H., T.T., R.N., M.S., T.T., and T.I. researched the data. K.T. wrote this manuscript. T.I. reviewed the manuscript. All authors read and approved the final manuscript. 


\section{References}

1. Wilding JPH (2014) The role of the kidneys in glucose homeostasis in type 2 diabetes: Clinical implications and therapeutic significance through sodium glucose co-transporter 2 inhibitors. Metabolism 63: 1228-1237.

2. Fujita Y, Inagaki N (2014) Renal sodium glucose cotransporter 2 as a novel therapeutic approach to treatment of type 2 diabetes: Clinical data and mechanism of action. J Diabetes Investig 5: 265-275.

3. Kanai Y, Lee WS, You G, Brown D, Hediger MA (1994) The human kidney low affinity $\mathrm{Na}+$ /glucose cotransporter SGLT2. Delineation of the major renal reabsorptive mechanism for D-glucose. J Clin Invest 93: 397-404.

4. Abdul-Ghani MA, Norton L, Defronzo RA (2011) Role of sodium-glucose cotransporter 2 (SGLT2) inhibitors in the treatment of type 2 diabetes. Endocr Rev 32: 515-531.

5. Kurosaki E, Ogasawara H (2013) Ipragliflozin and other sodium-glucose cotransporter-2 (SGLT2) inhibitors in the treatment of type 2 diabetes: preclinical and clinical data. Pharmacol Ther 139: 51-59.

6. Liang Y, Arakawa K, Ueta K, Matsushita Y, Kuriyama C, et al. (2012) Effect of canagliflozin on renal threshold for glucose, glycemia, and body weight in normal and diabetic models. PLos One 7: e30555.

7. Drozdowski LA, Thomson AB (2006) Intestinal sugar transport. World J Gastroenterol 12: 1657-1670.

8. Kanai Y, Stelzner M, Nussberger S, Khawaja S, Hebert SC, et al. (1994) The neuronal and epithelial human high affinity glutamate transporter. Insights into structure and mechanism of transport. J Biol Chem 269: 20599-20606.

9. Polidori D, Sha S, Mudaliar S, Ciaraldi TP, Ghosh A, et al. (2013) Canagliflozin lowers postprandial glucose and insulin by delaying intestinal glucose absorption in addition to increasing urinary glucose excretion: results of a randomized, placebo-controlled study. Diabetes Care 36: 2154-2161.

10. Oguma T, Nakayama K, Kuriyama C, Matsushita Y, Yoshida K, et al. (2015) Intestinal Sodium Glucose Cotransporter 1 Inhibition Enhances Glucagon-Like Peptide-1 Secretion in Normal and Diabetic Rodents. $J$ Pharmacol Exp Ther 354: 279-289.

11. Oguma T, Kuriyama C, Nakayama K, Matsushita Y, Yoshida K, et al. (2015) The effect of combined treatment with canagliflozin and teneligliptin on glucose intolerance in Zucker diabetic fatty rats. $J$ Pharmacol Sci 127: 456-461.
12. Dyer J, Wood IS, Palejwala A, Ellis A, Shirazi-Beechey SP (2002) Expression of monosaccharide transporters in intestine of diabetic humans. Am J Physiol Gastrointest Liver Physiol 282: G241-248.

13. Treatment guide for diabetes edited by Japan Diabetes Society 2007, Bunkodo Co., Ltd, Tokyo, Japan (In Japanese).

14. Rask E, Olsson T, Soderberg S, Johnson O, Seckl J, et al. (2001) Impaired incretin response after a mixed meal is associated with insulin resistance in nondiabetic men. Diabetes Care 24: 1640-1645.

15. Roberge JN, Brubaker PL (1993) Regulation of intestinal proglucagon-derived peptide secretion by glucosedependent insulinotropic peptide in a novel enteroendocrine loop. Endocrinology 133: 233-240.

16. Holst JJ (2007) The Physiology of glucacon-like peptide 1. Physiol Rev 87: 1409-1439.

17. Pais E, Gribble FM, Reimann F (2016) Stimulation of incretin secreting cells. Ther Adv Endocrinol Metab 7: 24-42.

18. Ferrannini E, Muscelli E, Frascerra S, Baldi S, Mari A, et al. (2014) Metabolic response to sodium-glucose cotransporter 2 inhibition in type 2 diabetic patients. $J$ Clin Invest 124: 499-508.

19. Sha S, Devineni D, Ghosh A, Polidori D, Chien S, et al. (2011) Canagliflozin, a novel inhibitor of sodium glucose co-transporter 2 , dose dependently reduces calculated renal threshold for glucose excretion and increases urinary glucose excretion in healthy subjects. Diabetes Obes Metab 13: 669-672.

20. Stein P, Berg JK, Morrow L, Polidori D, Artis E, et al. (2014) Canagliflozin, a sodium glucose co-transporter 2 inhibitor, reduces post-meal glucose excusion in patients with type 2 diabetes by a non-renal mechanism: results of a randomized trial. Metabolism 63: 1296-1303.

21. Lee EY, Kaneko S, Jutabha P, Zhang X, Seino S, et al. (2015) Distinct action of the $\alpha$-glucosidase inhibitor miglitol on SGLT3, enteroendocrine cells, and GLP1 secretion. J Endocrinol 224: 205-214.

22. Moriya R, Shirakura T, Ito J, Mashiko S, Seo T (2009) Activation of sodium-glucose cotransporter 1 ameliorates hyperglycemia by mediating incretin secretion in mice. Am J physiol Endocrinol Metab 297: E1358-1365.

23. Takebayashi K, Sakurai S, Suzuki T, Hori K, Terasawa T, et al. (2014) Effect of combination therapy with alogliptin and lansoprazole on glycemic control in patients with type 2 diabetes. Endocr J 61: 1031-1039. 